\title{
Counting King Permutations on the Cylinder
}

\author{
Eli Bagno, Estrella Eisenberg, Shulamit Reches, and Moriah Sigron \\ Jerusalem College of Technology,21 Havaad Haleumi, Jerusalem, Israel
Email: $\{$ bagnoe, estrella\}@g.jct.ac.il, shulamit.reches@gmail.com, moria.sigron@mail.huji.ac.il
}

Received: October 21, 2021, Accepted: January 24, 2022, Published: February 4, 2022

The authors: Released under the CC BY-ND license (International 4.0)

ABstRaCT: A permutation $\sigma=\sigma_{1} \cdots \sigma_{n} \in S_{n}$ is called a cylindrical king permutation if $\left|\sigma_{i+1}-\sigma_{i}\right|>1$ for each $1 \leq i \leq n-1$ and $\left|\sigma_{1}-\sigma_{n}\right|>1$. The name comes from the way one can see these permutations as describing locations of $n$ kings on a chessboard of order $n \times n$ in such a way that

- Each row and each column contains exactly one king.

- No two kings are attacking each other.

- A king can move off a certain row and reappear at the beginning of that row.

We present some results regarding the distribution of the cylindrical king permutations, including some interesting recursions. We also show that asymptotically, almost all the 'king' permutations are cylindrical king permutations, where a 'king' is a permutation that satisfies the first two of the three conditions above.

In the second part of this article, we investigate the poset of the cylindrical king permutations with respect to containment and its structure. We examine those cylindrical king permutations whose downset is as large as possible in the upper ranks. We use a modification of Manhattan distance of the plot of a permutation and some of its applications to the cylindrical context to find a criterion for such a permutation to be $k$-prolific. One of our main results is that for every two cylindrical king permutations, $\pi \in S_{k}$ and $\sigma \in S_{m}$ such that $\sigma$ covers $\pi$ in the sub-poset of cylindrical king permutations one has $m-k \leq 4$.

Keywords: Downset; Pattern; Pattern poset; Permutation; Permutation statistics; Prolific permutation

2020 Mathematics Subject Classification: 05A05; 06A07

\section{Introduction}

In a recent paper [5], we dealt with the set of king permutations which is the set of permutations $\pi=\pi_{1} \cdots \pi_{n} \in$ $S_{n}$ such that for each $1<i \leq n,\left|\pi_{i}-\pi_{i-1}\right|>1$. The inspiration was Hertzsprung's problem [16], dealing with the number of ways to arrange $n$ non-attacking chess kings on an $n \times n$ chessboard such that each column and each row contains exactly one chess king. There is a tight connection between the chess problem and the symmetric group $S_{n}$. If we consider a permutation $\pi=\pi_{1} \cdots \pi_{n} \in S_{n}$ in a geometrical way as its plot, i.e., the set of all lattice points of the form $\left(i, \pi_{i}\right)$ where $1 \leq i \leq n$, the problem of finding all the ways to arrange $n$ non-attacking chess kings is equivalent to the problem of finding all king permutations $\pi \in S_{n}$. This set is counted in OEIS A002464 [16].

Let $K_{n}$ be the set of all such permutations in $S_{n}$. For example, $K_{1}=S_{1}, K_{2}=K_{3}=\emptyset$, and $K_{4}=$ $\{3142,2413\}$. An explicit formula for the number of king permutations was given by Robbins [15]. He also showed that when $n \rightarrow \infty$, the probability of picking such a permutation from $S_{n}$ approaches $e^{-2}$. A nice generalization of the king problem is the dinner table problem, see for example [3] and [18].

A natural question is to extend Hertzsprung's problem to a celebrated variant of the chess game, namely the cylindrical chess, in which the right and left edges of the board are imagined to be joined in such a way that a piece can move off one edge and reappear at the opposite edge, moving in the same line [12].

Definition 1.1. Let $C K_{n}$ be defined as

$$
C K_{n}=\left\{\pi=\pi_{1} \cdots \pi_{n} \in S_{n}:\left|\pi_{i}-\pi_{i+1}\right|>1,\left|\pi_{1}-\pi_{n}\right|>1,1 \leq i \leq n-1\right\} .
$$

An element of $C K_{n}$ will be called a cylindrical king. 
For example, $C K_{1}=S_{1}, C K_{2}=C K_{3}=C K_{4}=\emptyset$ and

$$
C K_{5}=\{31425,14253,42531,25314,53142,24135,41352,13524,35241,52413\} .
$$

Note that each cylindrical king can also be seen as a directed Hamiltonian path (in the complement of the n-cycle graph). The sets $C K_{n}$ are counted by the sequence A002493 of OEIS [17]. The first 9 elements are $1,0,0,0,10,60,462,3920,36954$. They were counted by Abramson and Moser in [1]. See also some enumerations pertaining to this set in page 627 of [13]. We present some results regarding the distribution of the cylindrical king permutations, including some interesting recursions. We also show that asymptotically, almost all the 'king' permutations are cylindrical king permutations.

In the second part of this article, we investigate the poset of the cylindrical king permutations with respect to containment and its structure. We examine those cylindrical king permutations whose downset is as large as possible in the upper ranks. We use a modification of Manhattan distance of the plot of a permutation and some of its applications to the cylindrical context to find a criterion for such a permutation to be $k$-prolific. One of our main results is that for every two cylindrical king permutations, $\pi \in S_{k}$ and $\sigma \in S_{m}$ such that $\sigma$ covers $\pi$ in the sub-poset of cylindrical king permutations one has $m-k \leq 4$.

\section{General definitions and main results}

\subsection{Bonds and cyclic bonds}

In order to facilitate our counting of cylindrical king permutations we define a new concept: the cyclic bond, which extends the known concept of a bond appeared in $[10,11]$.

A bond in a permutation $\pi \in S_{n}$ is a length 2 consecutive sub-sequence of adjacent numbers (i.e. interval of order $2[6]$ ). Note that a king permutation is a permutation without bonds. This point of view can be used also to describe the set of cylindrical king permutations, provided that we slightly modify the definition of the bond in order to obtain what we call in this paper a cyclic bond.

Definition 2.1. Let $\pi=\pi_{1} \cdots \pi_{n} \in S_{n}$ and let $i \in[n-1]$. We say that the pair $\left(\pi_{i}, \pi_{i+1}\right)$ is a (regular) bond in $\pi$ if $\pi_{i}-\pi_{i+1}= \pm 1$. If $\pi_{n}-\pi_{1}= \pm 1$ then we may say that the pair $\left(\pi_{n}, \pi_{1}\right)$ is an edge bond of $\sigma$. In general, adopting the convention that $\pi_{n+1}=\pi_{1}$, we say that $\left(\pi_{i}, \pi_{i+1}\right)$ is a cyclic bond if it is a regular or an edge bond.

Example 2.1. In $\pi=41325$ there are 2 cyclic bonds. The regular bond (3,2) and the edge bond (5,4).

According to this new definition, a permutation is a cylindrical king if and only if it has no cyclic bonds. This type of modification from regular to cyclic parameters has been used also in the case of the descent parameter. See for example $[8,14]$.

Aside from its role in identifying the cylindrical king permutations, the definition of cyclic bonds leads to some interesting counting results by itself. For each $\pi \in S_{n}$ we denote by $\operatorname{bnd}(\pi)$ the number of regular bonds in $\pi$ and by $\operatorname{cbnd}(\pi)$ the number of cyclic bonds in $\pi$.

Definition 2.1. Let $B_{0}(t)=C B_{0}(t)=1$ and for $n \geq 1$ :

$$
B_{n}(t)=\sum_{\pi \in S_{n}} t^{b n d(\pi)}
$$

and

$$
C B_{n}(t)=\sum_{\pi \in S_{n}} t^{\operatorname{cbnd}(\pi)}
$$

$A$ simple calculation shows that $B_{1}(t)=C B_{1}(t)=1, B_{2}(t)=2 t, C B_{2}(t)=2 t^{2}, B_{3}(t)=2 t^{2}+4 t$ and $C B_{3}(t)=6 t^{2}$. Note that we chose to consider the permutations of $S_{2}$ as having 2 cyclic bonds each.

In Section 3 we present some results regarding the counting of cyclic bonds and their effect on the structure of the cylindrical king permutations. First we present some relations between the distribution of the number of regular bonds and the distribution of the number of cyclic bonds (Theorem 3.1 ) for $n \geq 2$ :

$$
C B_{n+1}(t)=(n+1) B_{n}(t)+2(n+1) \sum_{i=1}^{n}(t-1)^{i} B_{n-i}(t),
$$

where the initial conditions are as in Definition 2.1 .

We also have (Theorem 3.2) for $n \geq 1$ :

$$
B_{n}(t)=C B_{n}(t)+\frac{1}{n}(1-t) C B_{n}^{\prime}(t) .
$$




\section{$2.2 \quad$ Asymptotic results}

The results listed above have an impact on the structure of the cylindrical king permutations and thus they enable us to connect the number of king permutations to the number of cylindrical king permutations (Corollary 3.1): For $n \geq 2,\left|C K_{n+1}\right|=(n+1)\left|K_{n}\right|+2(n+1) \sum_{i=1}^{n}(-1)^{i}\left|K_{n-i}\right|$ while $\left|K_{0}\right|=1$.

Section 4 exhibits a recursion connecting the sizes of the sets of cylindrical kings and non-cylindrical king permutations, using a combinatorial proof (Theorem 4.1):

$$
\left|A_{n}\right|=2\left|K_{n-1}\right|+\left|A_{n-2}\right|, \text { where } A_{n}=K_{n} \backslash C K_{n} .
$$

This enables us to calculate the asymptotic ratio of the set of cylindrical king permutations in the entire set of the king permutations (Theorem 4.1):

The asymptotic value of $\left|C K_{n}\right| /\left|K_{n}\right|$ is equal to 1 .

\subsection{The distribution of cylindrical king permutations}

In Section 5 we calculate the distribution of cylindrical king permutations, using the distribution of cyclic bonds (Theorem 5.1 and Theorem 5.2).

\subsection{Prolific cyclic king permutations}

From a geometric point of view, we can recognize king permutations, as well as cylindrical king permutations, by considering the distances between the points of their plots. The Manhattan or taxicab distance is defined as follows.

Definition 2.2. Let $\sigma \in S_{n}$ and let $i, j \in[n]$. The (Manhattan) distance between the $i-t h$ and the $j-t h$ entries is defined to be the $L_{1}$ distance between the corresponding points in the plot of $\sigma$ :

$$
d_{\sigma}(i, j)=\left\|\left(i, \sigma_{i}\right)-\left(j, \sigma_{j}\right)\right\|_{1}=|i-j|+\left|\sigma_{i}-\sigma_{j}\right| .
$$

In $[2,7]$, the concept of breadth of a permutation $\sigma \in S_{n}$ is defined to be the minimal distance between any two distinct entries of $\sigma$. Explicitly:

$$
\operatorname{br}(\sigma)=\min _{i, j \in[n], i \neq j} d_{\sigma}(i, j) .
$$

Example 2.2. For $\sigma=5371426$ we have $d_{\sigma}(2,5)=4, d_{\sigma}(1,4)=7, d_{\sigma}(1,2)=3$ and its breadth is br $(\sigma)=3$

In order to characterize cylindrical king permutations in a similar way, we modify in Section 6 the concepts of (Manhattan) distance and breadth to cyclic (Manhattan) distance and cyclic breadth respectively.

For a permutation $\sigma \in S_{n}$ and $1 \leq k \leq n-1$, the maximal possible number of distinct permutations $\pi \in S_{n-k}$ which are contained in $\sigma$ is $\left(\begin{array}{l}n \\ k\end{array}\right)$. Indeed, such a permutation $\pi$, is obtained from $\sigma$ by deletion of $k$ entries from the one-line notation of $\sigma$. Following [2], our interest is in those permutations $\sigma \in C K_{n}$ which contain maximally many patterns $\pi \in C K_{n-k}$.

We are interested in characterizing the cylindrical king permutations which contain a maximal number of distinct cylindrical king patterns. To this aim, we use and modify the following concept of $k$-prolific permutation which was defined in [2]:

Definition 2.3. A permutation $\sigma \in S_{n}$ is called $k$-prolific if $\left|\left\{\pi \in S_{n-k} \mid \pi \prec \sigma\right\}\right|=\left(\begin{array}{l}n \\ k\end{array}\right)$.

In other words, a permutation $\sigma=\sigma_{1} \cdots \sigma_{n}$ is $k$-prolific if each $(n-k)$-subset of entries of $\sigma$ forms a unique pattern in $S_{n-k}$. It is known (see [5]) that $\sigma \in S_{n}$ is 1-prolific if and only if $\sigma$ is a king permutation, and this happens exactly when $b r(\sigma) \geq 3$.

In Section 6 we first prove that omitting a single entry from a permutation may decrease the cyclic breadth by at most one, and that for $\sigma \in C K_{n}$, there are $n$ distinct permutations $\sigma^{\prime} \in C K_{n-1}$ such that $\sigma^{\prime} \prec \sigma$ if and only if the cyclic breadth of $\sigma$ is greater than 3 (see Proposition 6.1 and Proposition 6.2 respectively). Then, using these propositions and our modification to the concept of $k$-prolific, we get generalizations of Theorem 2.25 in [2] to king permutations and cylindrical king permutations respectively:

1. A permutation $\sigma$ is $k$-prolific in $K_{n}$ if and only if its breadth is greater than or equal to $k+3$. (see Theorem 6.1).

2. A permutation $\sigma$ is $k$-prolific in $C K_{n}$ if and only if its cyclic breadth is greater or equal to $k+3$. (see Theorem 6.2). 


\subsection{The poset structure}

Another aspect of cylindrical king permutations is the structure of their poset. For $\sigma \in S_{n}$ and $\pi \in S_{k},(k<n)$, we say that $\sigma$ contains $\pi$ if there is a sub-sequence of (the one line notation of) $\sigma$ that is order-isomorphic to that of $\pi$. If this is the case, we write $\pi \prec \sigma$. In this study we are interested in the sub-poset $\mathbb{C K}=\bigcup_{n \geq 1} C K_{n}$ of the poset $\mathbb{S}=\bigcup_{n \geq 1} S_{n}$ with respect to the containment relation.

Here is an example of the downset of the cylindrical king permutation 5246173 in $\mathbb{C K}$ :

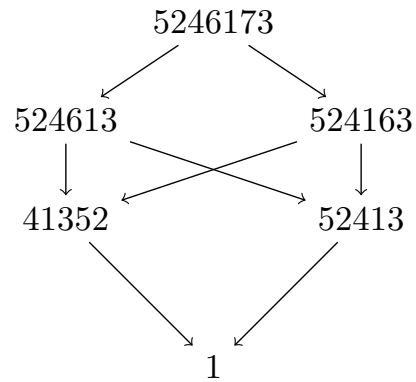

In Section 7 we investigate the structure of the poset of cylindrical king permutations. That section is a direct continuation of our previous paper [5], which discusses the structure of the poset of king permutations. Since the set of cylindrical king permutations is a distinguished subset of the set of king permutations, we examine which properties of the poset of the cylindrical king permutations have inherited from the kings poset and which features are different. We identify the building blocks of the poset of cylindrical king permutations:

- For every $\sigma \in C K_{n}(n \geq 5)$ there is some $\pi \in C K_{5}$ such that $\pi \preceq \sigma$ (see Theorem 7.1).

Another result is that if $\sigma \in C K_{n}$ covers $\pi \in C K_{m}$ in the poset $\mathbb{C K}$, then $n-m \leq 4$ :

- Let $\sigma \in C K_{m}$ and $\pi \in C K_{k}$ be such that $\pi \prec \sigma$ and $m-k>4$. Then there is some $\tau \in \mathbb{C K}$ such that $\pi \prec \tau \prec \sigma$ (see Theorem 7.2).

\subsection{General definitions}

Throughout this paper, we will make use of the following definitions.

Definition 2.4. For $\sigma \in S_{n}$ and $1 \leq i \leq n$ let $\nabla_{i}^{*}(\sigma)$ be the permutation in $S_{n-1}$ obtained from $\sigma$ by omitting the value $i$ and relabeling the rest of the values by the set $\{1, \ldots, n-1\}$ in an order preserving way (this is usually called standardization).

Occasionally we use the term "omitting the element $i$ from $\sigma$ " instead of the notation $\nabla_{i}^{*}(\sigma)$.

The following definition is inspired by definition 3 in [11].

Definition 2.5. For $\sigma \in S_{n}, 1 \leq i \leq n+1$ and $1 \leq j \leq n+1$ let $\Delta_{i}(\sigma, j)$ be the permutation $\tau \in S_{n+1}$ obtained from $\sigma$ by adding the value $i-\frac{1}{2}$ before place $j$ and relabeling the rest of the values by the set $\{1, \ldots, n, n+1\}$ in an order preserving way.

Occasionally we use the term "adding the element $i$ in the $j$-th place of sigma" instead of the notation $\Delta_{i}(\sigma, j)$.

For example if $\sigma=31425$ then $\nabla_{2}^{*}(\sigma)=2134$ and $\nabla_{3}^{*}(\sigma)=1324, \Delta_{4}(\sigma, 2)=341526$ and $\Delta_{1}(\sigma, 6)=425361$.

\section{Cyclic bonds and their effect on cylindrical king per- mutations}

\subsection{The number of regular bonds vs. the number of cyclic bonds}

Recall that for each $\pi \in S_{n}$ we denote by $\operatorname{bnd}(\pi)$ the number of regular bonds in $\pi$ and by $\operatorname{cbnd}(\pi)$ the number of cyclic bonds in $\pi$.

Let $C_{n}$ be the cyclic sub-group of $S_{n}$, generated by the cycle $\omega=(12 \cdots n)$. Note that the parameter cbnd is invariant under the right action of $C_{n}$ (left cyclic shift of the one-line notation of $\pi$ ). Explicitly:

$$
\operatorname{cbnd}(\pi)=\operatorname{cbnd}\left(\pi \omega^{i}\right) \text { for } 1 \leq i \leq n-1 .
$$


Example 3.1. For $n=4$ one can easily see that if $\pi=2134$ then $\pi \omega=1342, \pi \omega^{2}=3421$ and $\pi \omega^{3}=4213$. All of them have exactly 2 cyclic bonds.

In order to count the permutations in $S_{n+1}$ with exactly $k$ cyclic bonds, we count only those permutations whose last element is $n+1$, and multiply by $n+1$. Formally,

$$
\left|\left\{\pi \in S_{n+1}: \operatorname{cbnd}(\pi)=k\right\}\right|=(n+1) \mid\left\{\pi \in S_{n+1}: \operatorname{cbnd}(\pi)=k \text { and } \pi_{n+1}=n+1\right\} \mid
$$

Consider a permutation $\pi \in S_{n+1}$ such that $\pi_{n+1}=n+1$, and define $\pi^{\prime} \in S_{n}$ such that $\pi_{i}^{\prime}=\pi_{i}$ for $1 \leq i \leq n$. Then we have:

$$
\operatorname{cbnd}(\pi)= \begin{cases}\operatorname{bnd}\left(\pi^{\prime}\right)+1 & \text { if } \pi_{n}^{\prime}=n \text { or } \pi_{1}^{\prime}=n \\ \operatorname{bnd}\left(\pi^{\prime}\right) & \text { otherwise. }\end{cases}
$$

Now, we discuss the connection between the distributions of bonds and cyclic bonds (using Definition 2.1). For $n \geq 2$ let

$$
B_{n}^{1}(t)=\sum_{\pi \in S_{n}, \pi_{1}=n} t^{b n d(\pi)}
$$

and

$$
B_{n}^{n}(t)=\sum_{\pi \in S_{n}, \pi_{n}=n} t^{b n d(\pi)}
$$

If we denote

$$
C B_{n}^{n}(t)=\sum_{\pi \in S_{n}, \pi_{n}=n} t^{\operatorname{cbnd}(\pi)}
$$

then according to (2) we have:

$$
C B_{n+1}(t)=(n+1) C B_{n+1}^{n+1}(t) .
$$

Then we have by (3) for $n \geq 2$ :

$$
C B_{n+1}^{n+1}(t)=\left(B_{n}(t)-B_{n}^{1}(t)-B_{n}^{n}(t)\right)+t\left(B_{n}^{1}(t)+B_{n}^{n}(t)\right) .
$$

Indeed $B_{n}^{1}(t)=B_{n}^{n}(t)$ and thus

$$
C B_{n+1}^{n+1}(t)=B_{n}(t)-2 B_{n}^{1}(t)+2 t B_{n}^{1}(t)
$$

and according to (4) and (6):

$$
\begin{aligned}
C B_{n+1}(t) & =(n+1)\left[B_{n}(t)-2 B_{n}^{1}(t)+2 t B_{n}^{1}(t)\right] \\
& =(n+1) B_{n}(t)+2(n+1)(t-1) B_{n}^{1}(t) .
\end{aligned}
$$

In addition for $n \geq 2$ :

$$
B_{n}^{1}(t)=\left(B_{n-1}(t)-B_{n-1}^{1}(t)\right)+t B_{n-1}^{1}(t)=B_{n-1}(t)+(t-1) B_{n-1}^{1}(t),
$$

where $B_{1}^{1}(t)=1$. A simple induction will show that for $n \geq 2$ :

$$
B_{n}^{1}(t)=\sum_{i=1}^{n}(t-1)^{i-1} B_{n-i}(t)
$$

Using (7) we have for $n \geq 2$ :

$$
C B_{n+1}(t)=(n+1) B_{n}(t)+2(n+1)(t-1) B_{n}^{1}(t)=(n+1) B_{n}(t)+2(n+1)(t-1) \sum_{i=1}^{n}(t-1)^{i-1} B_{n-i}(t) .
$$

According to the above calculations we obtain the following theorem.

Theorem 3.1. Let $n \geq 2$. Then

$$
C B_{n+1}(t)=(n+1) B_{n}(t)+2(n+1) \sum_{i=1}^{n}(t-1)^{i} B_{n-i}(t),
$$

where the initial conditions are as in Definition 2.1.

In Theorem 3.1 we expressed the generating function of the cyclic bonds in terms of the regular bonds of lower levels. The next theorem presents $B_{n}(t)$ directly in terms of $C B_{n}(t)$ and its derivative. 
Theorem 3.2. Let $n \geq 1$. Then we have

$$
B_{n}(t)=C B_{n}(t)+\frac{1}{n}(1-t) C B_{n}^{\prime}(t)
$$

Proof. Let $\pi \in S_{n}$ be such that $\operatorname{cbnd}(\pi)=k$, and denote by $[\pi]$ the orbit of $\pi$ under the right action of $C_{n}$. The contribution of $[\pi]$ to the polynomial $C B_{n}(t)$ is $n t^{k}$, and thus its contribution to the right hand side is $n t^{k}+(1-t) k t^{k-1}$. In order to complete the proof, we have to find the contribution of $[\pi]$ to $B_{n}(t)$. Writing $\pi=\pi_{1} \cdots \pi_{n}$, each representative of its orbit starts with some $\pi_{i}, 1 \leq i \leq n$. The number of regular bonds in $\pi_{i} \pi_{i+1} \cdots \pi_{1} \cdots \pi_{i-1}$ is $k-1$ if $\left(\pi_{i-1}, \pi_{i}\right)$ is a cyclic bond and $k$ otherwise. We conclude that the contribution of $[\pi]$ to $B_{n}(t)$ is exactly $k t^{k-1}+(n-k) t^{k}=n t^{k}+(1-t) k t^{k-1}$ as required.

Combining Theorems 3.1, and 3.2, we can create a recursion for the generating function of the cyclic bonds based only on terms of cyclic bonds only.

Theorem 3.3. Let $n \geq 2$. Then

$$
C B_{n+1}(t)=(n+1)\left[C B_{n}(t)+2 \sum_{i=1}^{n}(t-1)^{i} C B_{n-i}(t)\right]+\frac{n+1}{n}(1-t)\left[C B_{n}^{\prime}(t)+2 \sum_{i=1}^{n}(t-1)^{i} C B_{n-i}^{\prime}(t)\right],
$$

where the initial conditions are as in Definition 2.1.

\subsection{The number of kings vs. the number of cylindrical king permutations}

Recall that a king permutation is actually a permutation without bonds. As a result, we get that $\left|K_{n}\right|=B_{n}(0)$. Moreover, a permutation is a cylindrical king if and only if it has no cyclic bonds, thus $\left|C K_{n}\right|=C B_{n}(0)$. By Theorem 3.1, we have the following corollary.

Corollary 3.1. For $n \geq 2$,

$$
\left|C K_{n+1}\right|=(n+1)\left|K_{n}\right|+2(n+1) \sum_{i=1}^{n}(-1)^{i}\left|K_{n-i}\right|
$$

while $\left|K_{0}\right|=1$.

\section{Another recursion and the asymptotic value of $\frac{\left|C K_{n}\right|}{\left|K_{n}\right|}$}

In this section, we introduce another recursion connecting the sizes of the sets of cylindrical king permutations and non-cylindrical king permutations and present a combinatorial proof for this recursion. Eventually, this will allow us to prove that the proportion of the set of cylindrical king permutations in the entire set of king permutations is asymptotically 1 .

First, define for each $n \geq 1$ the set of king permutations which are not cylindrical:

$$
A_{n}=K_{n} \backslash C K_{n}
$$

Theorem 4.1. We have the following recursion for each $n \geq 2$ :

$$
\left|A_{n}\right|=2\left|K_{n-1}\right|+\left|A_{n-2}\right| \text {. }
$$

Proof. The recursion can be verified by brute force for $n=2$ and $n=3$, then for $4 \leq n$, every permutation $\pi \in A_{n}$ can be written in one of the following forms:

- $A_{n}^{0}: \pi=k, k+2, \cdots, k-1, k+1$ or its reverse for $2 \leq k \leq n-2$.

- $A_{n}^{1}: \pi=k, l, \ldots, k-1, k+1$ or its reverse for $l \neq k+2$ and $2 \leq k \leq n-1$.

- $A_{n}^{2}: \pi=k, k+2, \ldots, m, k+1$ or its reverse for $m \neq k-1$ and $1 \leq k \leq n-2$.

- $A_{n}^{3}: \pi=k, l, \ldots, m, k+1$ or its reverse where $m \neq k-1, l \neq k+2$ and $1 \leq k \leq n-1$.

This is a partition of the set $A_{n}$ into the 4 disjoint sets $A_{n}^{i}$ for $0 \leq i \leq 3$.

Throughout this proof, we use the terms "omitting" and "adding" according to Definitions 2.4 and 2.5.

We define the following maps: 
1. A bijection $f_{0}: A_{n}^{0} \rightarrow A_{n-2}$ which implies that $\left|A_{n}^{0}\right|=\left|A_{n-2}\right|$.

2. Two bijections $f_{1}: A_{n}^{1} \rightarrow A_{n-1}$ and $f_{2}: A_{n}^{2} \rightarrow A_{n-1}$ which imply that $\left|A_{n}^{1}\right|=\left|A_{n-1}\right|=\left|A_{n}^{2}\right|$.

3. A 2 to 1 mapping $f_{3}: A_{n}^{3} \rightarrow C K_{n-1}$, which implies that $2\left|C K_{n-1}\right|=\left|A_{n}^{3}\right|$.

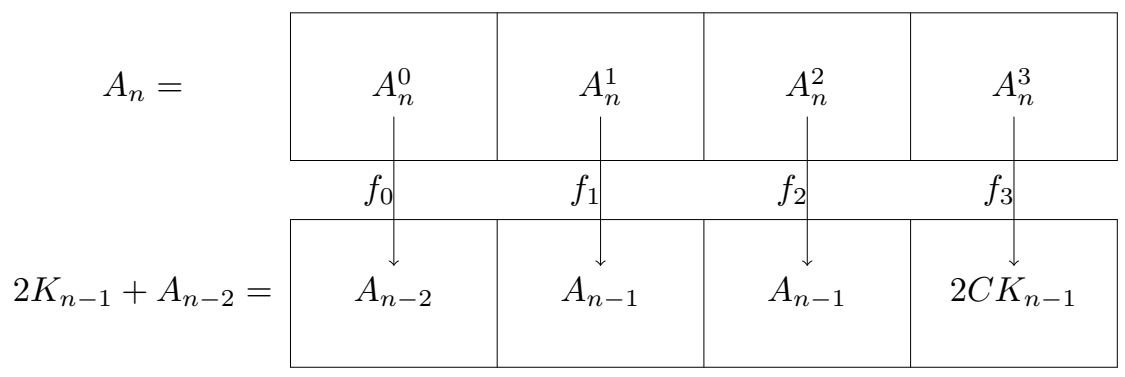

We start by defining a bijection $f_{0}: A_{n}^{0} \rightarrow A_{n-2}$ in the following way: $f_{0}(\pi)=\nabla_{k}^{*}\left(\nabla_{k+1}^{*}(\pi)\right)$.

For example, let $\pi=426153 \in A_{6}^{0}$. Then $f_{0}(\pi)=2413 \in A_{4}$.

In order to show that $f_{0}$ is bijective, we present the inverse function. Explicitly, for $\sigma=a-1 \cdots a \in A_{n-2}$ (or $\left.\sigma=a \cdots a-1 \in A_{n-2}\right) f_{0}^{-1}(\sigma)$ is obtained from $\sigma$ by adding the element $a$ sequentially at the two sides of $\sigma$, first to the side of $a-1$ and then to the other side.

For example, let $\sigma=2413$. Then we first add 3 to the left of $\sigma$ to get $\sigma^{\prime}=32514$ and then add again 3 to the right of $\sigma^{\prime}$ to get $426153 \in A_{n}^{0}$.

Next, we construct a function $f_{1}: A_{n}^{1} \rightarrow A_{n-1}$ by omitting from $\pi=\pi_{1} \cdots \pi_{n}$ the element $\max \left\{\pi_{1}, \pi_{n}\right\}$. For example: Let $\pi=5137246$, then $f_{1}(\pi)=513624$.

In a similar way, define $f_{2}: A_{n}^{2} \rightarrow A_{n-1}$ by omitting the element $\min \left\{\pi_{1}, \pi_{n}\right\}$. For instance, $f_{2}(5724136)=$ 624135.

Now, we show the inverse of the function $f_{1}$. First, if $\sigma=a \cdots a-1 \in A_{n-1}$, then $f_{1}^{-1}(\sigma)=a, l \ldots, a-1, a+$ $1 \in A_{n}^{1}$ (note that $l \neq a+2$ since otherwise $\sigma \notin A_{n-1}$ ). If $\sigma=a-1 \cdots a$ then $f_{1}^{-1}(\sigma)=a+1, a-1, \ldots, l, a \in A_{n}^{1}$, $(l \neq a+2)$.

Similarly, we show the inverse of $f_{2}$. First, if $\sigma=a \cdots a-1 \in A_{n-1}$, then $f_{2}^{-1}(\sigma)=a-1, a+1 \ldots l, a \in A_{n}^{2}$ (note that $l \neq a-2$ ). If $\sigma=a-1 \cdots a$ then $f_{2}^{-1}$ is obtained by adding $a-1$ at the end of $\sigma$, i.e. $f_{2}^{-1}(\sigma)=$ $a, l \ldots, a+1, a-1 \in A_{n}^{2}$ (since $l \neq a-2$ ).

Next, we construct a mapping $f_{3}: A_{n}^{3} \rightarrow C K_{n-1}$ which is 2 to 1 , i.e. each element of $C K_{n-1}$ will have exactly two preimages. For $\pi \in A_{n}^{3}$, the function $f_{3}$ omits from $\pi$ the maximum of its two extreme elements.

For example, let $\pi=364152 \in A_{6}^{3}$. Then $f_{3}(\pi)=53142 \in C K_{5}$. Note that we also have that $f_{3}(531426)=$ 53142. In order to see that the function $f_{3}$ is indeed 2 to 1 , we show how to go back from an arbitrary element of $C K_{n-1}$ to its two preimages. Let $\sigma=a \cdots b \in C K_{n-1}$, then we have that $a \neq b \pm 1$, so we define $\pi$ to be the permutation obtained by adding $a+1$ after $b$ and let $\pi^{\prime}$ be the permutation obtained by adding $b+1$ before $a$. It is easy to see then that $f_{3}(\pi)=f_{3}\left(\pi^{\prime}\right)=\sigma$.

From this data we have that

$$
\left|A_{n}\right|=\left|A_{n}^{0}\right|+\left|A_{n}^{1}\right|+\left|A_{n}^{2}\right|+\left|A_{n}^{3}\right|=\left|A_{n-2}\right|+2\left|A_{n-1}\right|+2\left|C K_{n-1}\right|=\left|A_{n-2}\right|+2\left|K_{n-1}\right| .
$$

Using the above theorem we can prove the following conclusion.

Corollary 4.1. The asymptotic value of $\left|C K_{n}\right| /\left|K_{n}\right|$ is equal to 1

Proof. By Theorem 4.1 we have that $\left|A_{n}\right|=2\left|K_{n-1}\right|+\left|A_{n-2}\right|$.

Thus, $\left|K_{n}\right|-\left|C K_{n}\right|=2\left|K_{n-1}\right|+\left|K_{n-2}\right|-\left|C K_{n-2}\right|$ and we obtain:

$$
\frac{\left|C K_{n}\right|}{\left|K_{n}\right|}=1-\frac{2\left|K_{n-1}\right|}{\left|K_{n}\right|}-\frac{\left|K_{n-2}\right|}{\left|K_{n}\right|}+\frac{\left|C K_{n-2}\right|}{\left|K_{n}\right|} .
$$

According to Robbins [15], when $n$ tends to infinity, the probability of picking a king permutation from $S_{n}$ approaches $e^{-2}$. Thus, the asymptotic value of $\left|K_{n}\right|$ is $n ! e^{-2}$ and thus the asymptotic value of $\frac{\left|K_{n-1}\right|}{\left|K_{n}\right|}$ is $\frac{1}{n} \rightarrow 0$ and the asymptotic value of $\frac{\left|K_{n-2}\right|}{\left|K_{n}\right|}$ is $\frac{1}{n^{2}} \rightarrow 0$. As a result,

$$
0 \leq \frac{\left|C K_{n-2}\right|}{\left|K_{n}\right|} \leq \frac{\left|K_{n-2}\right|}{\left|K_{n}\right|}
$$

Since we know that $\lim _{n \rightarrow \infty} \frac{\left|K_{n-2}\right|}{\left|K_{n}\right|}=0$, we conclude that $\lim _{n \rightarrow \infty} \frac{\left|C K_{n-2}\right|}{\left|K_{n}\right|}=0$, and therefore $\frac{\left|C K_{n}\right|}{\left|K_{n}\right|} \rightarrow 1$ 


\section{The distribution of cylindrical king permutations}

\subsection{The distribution of cyclic bonds}

In [11], Homberger calculated the distribution of the number of bonds. He used it, inter alia, to evaluate the number of king permutations, while considering them as permutations without bonds. In this section, we introduce the distribution of our new concept, the cyclic bonds, and use it to calculate the distribution of the cylindrical king permutations. In our recent paper [4] the concept of marked permutations is used. Here, we use again this concept in order to calculate the distribution of the cyclic bonds over $S_{n}$. We start with the following definition.

Definition 5.1. A marked permutation is a permutation $\pi \in S_{n}$ such that each cyclic bond is either marked or not. If several consecutive cyclic bonds are marked, then they form a run. An entry that is not marked is considered to be a run of length 1 (a trivial run). Note that a cyclic run of a permutation might be ascending or descending.

Note that each marked permutation $\pi \in S_{n}$ can be presented as a concatenation of runs. In order to identify the runs of a marked permutation, we put each run between two delimiters. This is done in a cyclic way. For example, the permutation 83124567 contributes the marked permutation $8|3| 12 \mid 4567$ (which consists of the three runs: $3,12,45678$ ), the marked permutation $8|3| 12|4567|$ (which consists of the four runs: $8,3,12,4567$ ), as well as the marked permutation $8|3| 1|2| 4567 \mid$ (which consists of the five runs: $8,3,1,2,4567$ ) and many more. Moreover, a marked permutation $\pi \in S_{n}$ with $m$ runs can be uniquely characterized by the following data: a vector containing the lengths of the runs (which is a composition $\lambda$ of $n$ ) and their directions (increasing or decreasing), a permutation $\sigma \in S_{m}$ which determines their locations and the number $r$ of places the last run occupies at the beginning of the permutation $\pi$. This idea will be best explained by an example.

Example 5.1. Let $\pi=2|45| 6|1| 987 \mid 3$. We write $\pi$ as a triple consisting of a 'directed' composition of $m=5$ parts $\lambda$, a permutation $\sigma \in S_{5}$ and $r=1$. First, write $\pi$ as a sequence of runs: $b_{1}=1, b_{2}=32, b_{3}=45, b_{4}=$ $6, b_{5}=987$. Each run contributes its length to the composition. Then for each part, we add the sign $\uparrow$ if the corresponding run is increasing, the sign $\downarrow$ if the run is decreasing and no arrow if the run is of length 1 . In our case we get $\lambda=(1,2 \downarrow, 2 \uparrow, 1,3 \downarrow)$. Now, $\sigma \in S_{5}$ is the permutation induced by the order of the blocks. In our case, since the block $b_{1}=1$ is located as the third block of $\pi$, we get $\sigma(3)=1$, (note that the digit 2 does not constitute a separate block but a part of the last block 32). Likewise, $b_{2}=3$ is located as the fifth block of $\pi$, so $\sigma(5)=2$ etc. We have: $\sigma=34152$. The marked permutation $\pi$ is now uniquely defined by the triple $(\lambda, \sigma, r)$. Note that the marked permutation $\tau=45|6| 1|987| 32 \mid$ has the same data except that now $r=0$.

In order to calculate the distribution of the number of cyclic bonds, we count marked permutations and use the inclusion-exclusion principle to obtain the desired distribution.

The next theorem presents a generating function for the number of cyclic bonds.

For each $k, n \geq 0$, we denote

$$
a_{n, k}=\left|\left\{\pi \in S_{n} \mid \operatorname{cbnd}(\pi)=k\right\}\right| .
$$

Furthermore, let

$$
H(z, u)=\sum_{n \geq 1} \sum_{k \geq 0} a_{n, k} z^{n} u^{k}
$$

be the generating function of the number of cylic bonds. We have now:

Theorem 5.1.

$$
H(z, u)=2 z^{2}(u-1)^{2}+\sum_{m \geq 1} m ! z^{m}\left(\frac{1+z(u-1)}{1-z(u-1)}\right)^{m-1} \frac{1+2 z(u-1)-z^{2}(u-1)^{2}}{1-2 z(u-1)+z^{2}(u-1)^{2}}
$$

Proof. We first calculate the generating function of marked cyclic bonds. Let

$$
F(z, u)=\sum_{n \geq 1} \sum_{k \geq 0} f_{n, k} z^{n} u^{k}
$$

where $f_{n, k}$ is the number of permutations of order $n$ with $k$ marked cyclic bonds. We count the marked permutations by considering each run separately. Let $m$ be the number of runs in a permutation. There are $m$ ! ways to arrange the runs in each marked permutation. For each such arrangement, we distinguish between the last run of a permutation and the other runs (if there is only one run then it will be considered the last one). We start with the first $m-1$ runs. Each run of length one contributes $z$, while each run of length $k \geq 2$ has $k-1$ regular bonds and can be increasing or decreasing, so it contributes $2 z^{k} u^{k-1}$. This gives us $\left(z+2 z^{2} u+2 z^{3} u^{2}+2 z^{4} u^{3}+\ldots\right)^{m-1}$. 
Now we discuss the last run that starts at the end of the permutation and may also emerge at the beginning of the permutation (like the run 1234 in the permutation $4|67| 5 \mid \mathbf{1 2 3}$ ). If it is of length 1 then it contributes $z$. If it has length $k \geq 2$ then it has $k-1$ cyclic bonds and may be decreasing or increasing. Moreover, its $k-r$ first digits are placed at the end of the permutation, and the other $r$ digits at the beginning of the permutation for $0 \leq r \leq k-1$. Thus its contribution is $2 k z^{k} u^{k-1}$. This gives us $\left(z+2 \cdot 2 z^{2} u+2 \cdot 3 z^{3} u^{2}+2 \cdot 4 z^{4} u^{3}+\ldots\right)$. This leads to

$$
\begin{aligned}
F(z, u) & =\sum_{m \geq 1} m !\left(z+2 z^{2} u+2 z^{3} u^{2}+2 z^{4} u^{3}+\ldots\right)^{m-1}\left(z+4 z^{2} u+6 z^{3} u^{2}+8 z^{4} u^{3}+\ldots\right) \\
& =\sum_{m \geq 1} m ! z^{m-1}\left(\frac{1+z u}{1-z u}\right)^{m-1}\left(z+\frac{2 z\left(2 z u-(z u)^{2}\right)}{(1-z u)^{2}}\right) \\
& =\sum_{m \geq 1} m ! z^{m}\left(\frac{1+z u}{1-z u}\right)^{m-1} \frac{1+2 z u-z^{2} u^{2}}{1-2 z u+z^{2} u^{2}} .
\end{aligned}
$$

Now, we can use $F(z, u)$ to obtain $H(z, u)$. Since $F(z, u)$ counts the marked cyclic bonds and $H(z, u)$ counts every cyclic bond, using the inclusion-exclusion principle it follows that $H(z, u)=F(z, u-1)$.

Using the above technique, we actually overcount the marked permutations 12 and 21 in $F$. Explicitly, $1|2|, 2|1|, 12|, 1| 2,21 \mid$ and $2 \mid 1$ contribute $z^{2}, z^{2}, z^{2} u, z^{2} u, z^{2} u$ and $z^{2} u$ respectively so that the contribution to $H(z, u)=F(z, u-1)$ is $4 z^{2} u-2 z^{2}$.

On the other hand, according to Definition 2.1, we consider 12 and 21 as having two cyclic bonds each, and so we expect them to contribute only $2 z^{2} u^{2}$ to $H(z, u)$. Hence, we have to add $2 z^{2}(u-1)^{2}$. Thus:

$$
H(z, u)=2 z^{2}(u-1)^{2}+\sum_{m \geq 1} m ! z^{m}\left(\frac{1+z(u-1)}{1-z(u-1)}\right)^{m-1} \frac{1+2 z(u-1)-z^{2}(u-1)^{2}}{1-2 z(u-1)+z^{2}(u-1)^{2}}
$$

\subsection{The distribution of cylindrical king permutations}

Substituting $u=0$ in Theorem 5.1 we can easily find a generating function for cylindrical king permutations, i.e., a function of the form $C K(z)=\sum_{n \geq 1}\left|C K_{n}\right| z^{n}$.

Theorem 5.2.

$$
C K(z)=2 z^{2}+\sum_{m \geq 1} m ! z^{m}\left(\frac{1-z}{1+z}\right)^{m-1}\left(\frac{\left(1-2 z-z^{2}\right)}{(1+z)^{2}}\right)
$$

Note that the corresponding formula for $K(z)$ is due to Carlitz (see [18] pp. 6-7).

\section{Cyclic breadth and $k$-prolific cylindrical king permu- tations}

We are interested in characterizing the cylindrical king permutations and analyzing some properties of their downsets in $\mathbb{C} \mathbb{K}$. In particular, in this section, we focus on those cylindrical king permutations which contain as many distinct patterns as possible in the poset $\mathbb{C} \mathbb{K}$. There is a close relationship between the breadth of a permutation and the number of distinct patterns it contains. Moreover, as mentioned above, one way to identify a king permutation is by verifying that its breadth is greater than or equal to 3 . In order to be able to identify cylindrical king permutations in a similar way, and especially those whose downsets in $\mathbb{C} \mathbb{K}$ have maximal size, we modify the definitions of the (Manhattan) distance and the breadth, introducing the cyclic (Manhattan) distance and the cyclic breadth of a permutation $\sigma \in S_{n}$.

Throughout this section we consider a permutation $\sigma=\sigma_{1} \cdots \sigma_{n}$ as if it was located on a circle in such a way that $\sigma_{1}$ occupies the topmost point of the circle (see Figure 1).

Definition 6.1. For $1 \leq i<j \leq n$, we denote by $((i, j))$ the shortest path on the circle leading from $i$ to $j$. We also set $\|j-i\|=|((i, j))|$, i.e. $\|j-i\|=\min (j-i, n-j+i)$.

Definition 6.2. Let $\sigma \in S_{n}$ and let $i<j \in[n]$. Let the cyclic (Manhattan) distance between the $i-$ th and the $j-$ th entries be defined as:

$$
c d_{\sigma}(i, j)=\left|\sigma_{j}-\sigma_{i}\right|+\|j-i\|
$$


The cyclic breadth of $\sigma \in S_{n}$ is then defined to be:

$$
\operatorname{cbr}(\sigma)=\min _{i, j \in[n], i \neq j} c d_{\sigma}(i, j) .
$$

Example 6.1. Let $\sigma=724915836 \in S_{9}$. Then $c d_{\sigma}(1,2)=(7-2)+\|2-1\|=6$, while cd $_{\sigma}(1,9)=(7-6)+$ $\|9-1\|=2$. The breadth of $\sigma$ is $\operatorname{cbr}(\sigma)=2$

We compare now between the regular (Manhattan) distance and the cyclic (Manhattan) distance as follows:

Observation 6.1. - For each $\sigma \in S_{n}$ and for each $i, j \in[n], \operatorname{cd}(i, j) \leq d_{\sigma}(i, j)$ and $\operatorname{so} \operatorname{cbr}(\sigma) \leq b r(\sigma)$.

- For every $i, j \in[n]$, we have $d_{\sigma}(i, j)=d_{\sigma^{-1}}\left(\sigma_{i}, \sigma_{j}\right)$ and thus br $(\sigma)=\operatorname{br}\left(\sigma^{-1}\right)$ which means that the regular (Manhattan) breadth is invariant under inversion.

- The cyclic breadth $\operatorname{cbr}(\sigma)$ is not always equal to $\operatorname{cbr}\left(\sigma^{-1}\right)$. For example, if $\sigma=72415836 \in S_{8}$ then $\sigma^{-1}=42735816$, so that $\operatorname{cbr}(\sigma)=c d_{\sigma}(1,8)=2$ but $\operatorname{cbr}\left(\sigma^{-1}\right)=c d_{\sigma^{-1}}(1,2)=3$.

Using our new definitions, we can identify the cylindrical king permutations as follows.

Observation 6.2. A permutation $\sigma$ of order $n>1$ is a cylindrical king permutation if and only if its cyclic breadth is greater than or equal to 3 .

Omitting a single entry from a permutation $\sigma \in S_{n}$ (and then standardising) produces a permutation $\sigma^{\prime} \in S_{n-1}$ such that $\sigma^{\prime} \prec \sigma$. In Proposition 2.24 of [2], Bevan and Homberger proved a proposition that partially controls the change in breadth of permutations in $S_{n}$ : omitting a single entry from a permutation decreases the breadth by at most one. We now present and prove a proposition that extends the above result, considering the cyclic breadth rather than the regular breadth. This enables us to characterize the cyclic breadth of permutations $\sigma^{\prime}$ which are contained in a certain permutation $\sigma$.

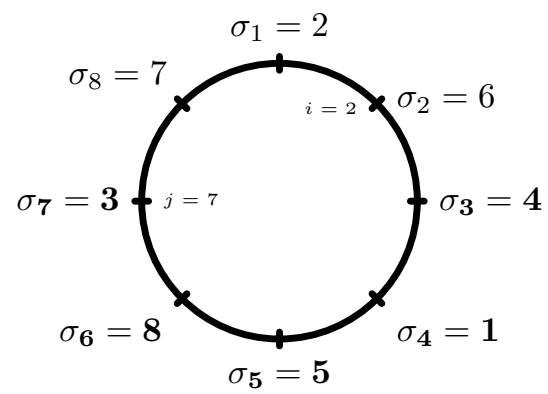

Figure 1: $\sigma=26415837$ located on a circle in such a way that $\sigma_{1}$ occupies the topmost point of the circle. $c d_{\sigma}(2,7)=|6-3|+|| 7-2||=6$ where $\|7-2\|=\min \{7-2,8+2-7\}$.

Proposition 6.1. Omitting a single entry from a permutation may decrease the cyclic breadth by at most one.

Proof. Let $\sigma \in S_{n}$ and let $1 \leq i<j \leq n$ be such that without loss of generality $\sigma_{i}<\sigma_{j}$. Recall that $c d_{\sigma}(i, j)$ is composed of the position part $\|j-i\|$ and the value part $\left|\sigma_{j}-\sigma_{i}\right|$. Let $\sigma^{\prime}$ be the permutation obtained from $\sigma$ by omitting an element $\sigma_{k}$ for some $k \notin\{i, j\}$. We discuss the effect of omitting $\sigma_{k}$ on each one of the parts of $c d_{\sigma}(i, j)$.

Observe that if $\sigma_{k}>\sigma_{j}$ or $\sigma_{k}<\sigma_{i}$ then this omission does not affect the value part of $c d_{\sigma}(i, j)$. On the other hand, if $\sigma_{i}<\sigma_{k}<\sigma_{j}$ then, its omission decreases the value part of $c d_{\sigma}(i, j)$ by 1 .

Similarly, the omission of $\sigma_{k}$ affects the position part if and only if $k \in((i, j))$. In case there is an effect, the difference is 1 .

We conclude, that the omission of $\sigma_{k}$ decreases $c d_{\sigma}(i, j)$ of $\sigma$ by 2 if and only if $\sigma_{i}<\sigma_{k}<\sigma_{j}$ and $k \in((i, j))$, in all other cases the difference is 0 or 1.

Now, let $i<j$ be such that $\operatorname{cbr}(\sigma)=c d_{\sigma}(i, j)$. Assume to the contrary that there is $k \in[n]$ such that $\operatorname{cbr}\left(\sigma^{\prime}\right)=\operatorname{cbr}(\sigma)-2$, where $\sigma^{\prime}$ is obtained from $\sigma$ by omitting $\sigma_{k}$. This means that $k \in((i, j))$ and $\sigma_{i}<\sigma_{k}<\sigma_{j}$. We have now:

$$
\operatorname{cbr}(\sigma) \leq c d_{\sigma}(i, k)=\sigma_{k}-\sigma_{i}+\|k-i\|<\sigma_{j}-\sigma_{i}+\|j-i\|=c d_{\sigma}(i, j),
$$

a contradiction.

Finally, we show that for $\operatorname{cbr}(\sigma)=c d_{\sigma}(i, j)$, an omission of one of the elements $\sigma_{i}$ or $\sigma_{j}$ also decreases the cyclic breadth by at most one. Without loss of generality let us consider the omission of $\sigma_{j}$.

Indeed, for every $m, l \neq j$ we have $\operatorname{cbr}(\sigma)=c d_{\sigma}(i, j) \leq c d_{\sigma}(m, l)$. If $c d_{\sigma}(i, j)<c d_{\sigma}(m, l)$ then the omission of $\sigma_{j}$ decreases $c d_{\sigma}(m, l)$ by at most 2 to a value that is greater or equal to $c d_{\sigma}(i, j)-1$. If $c d_{\sigma}(i, j)=c d_{\sigma}(m, l)$ then $\operatorname{cbr}(\sigma)=c d_{\sigma}(m, l)$, and due to the previous discussion, the omission of $\sigma_{j}$ decreases $c d_{\sigma}(m, l)$ by at most one. Therefore if $\sigma^{\prime}$ is obtained from $\sigma$ by omitting $\sigma_{j}$ then also $\operatorname{cbr}\left(\sigma^{\prime}\right) \geq \operatorname{cbr}(\sigma)-1$. 
Remark 6.1. Note that omitting an element $\sigma_{k}$ might also increase the breadth of a permutation $\sigma$. For example if $\sigma=351246$ then $\operatorname{cbr}(\sigma)=c d(3,4)=2$. If we omit $\sigma_{3}=1$, we get $\sigma^{\prime}=24135$ with $\operatorname{cbr}\left(\sigma^{\prime}\right)=3$.

In [9], Coleman demonstrates that maximizing the distance between every two elements in a permutation tends to increase the number of distinct sub-permutations (patterns).

The next proposition characterizes the permutations $\sigma \in C K_{n}$ which contain $n$ different patterns $\sigma^{\prime} \prec \sigma$ such that $\sigma^{\prime} \in C K_{n-1}$.

Proposition 6.2. For $\sigma \in C K_{n}$, there are $n$ distinct permutations $\sigma^{\prime} \in C K_{n-1}$ such that $\sigma^{\prime} \prec \sigma$ if and only if $\operatorname{cbr}(\sigma)>3$.

Proof. Let $\sigma \in C K_{n}$ and suppose that there are $n$ distinct permutations $\sigma^{\prime} \in C K_{n-1}$ such that $\sigma^{\prime} \prec \sigma$, which means that by omitting any entry of $\sigma$, we get a cylindrical king permutation. Since $\sigma \in C K_{n}$, according to Observation 6.2, $\operatorname{cbr}(\sigma) \geq 3$. Assume to the contrary that $\operatorname{cbr}(\sigma)=3$, thus there are entries $i$ and $j, i<j$ such that $\operatorname{cbr}(\sigma)=c d_{\sigma}(i, j)=3$, and hence that ||$j-i \|=1$ and $\left|\sigma_{j}-\sigma_{i}\right|=2$ or vice versa. Assume without loss of generality that $\sigma_{i}<\sigma_{j}$.

- If $\|j-i\|=1$ and $\sigma_{j}-\sigma_{i}=2$ then by omitting the value $\sigma_{i}+1$ we get $\sigma^{\prime}=\nabla_{\sigma_{i}+1}^{*}(\sigma) \notin C K_{n-1}$, a contradiction.

- If $\|j-i\|=2$ and $\sigma_{j}-\sigma_{i}=1$ then by omitting the value located between $\sigma_{i}$ and $\sigma_{j}, \sigma_{i+1}$, we get $\sigma^{\prime}=\nabla_{\sigma_{i+1}}^{*}(\sigma) \notin C K_{n-1}$, a contradiction.

Now, suppose that $\operatorname{cbr}(\sigma)>3$. By corollary 7 of [11], a permutation $\sigma \in S_{n}$ has maximum number of patterns $\sigma^{\prime} \prec \sigma$ with $\sigma^{\prime} \in S_{n-1}$, if and only if it has no bonds. The permutation $\sigma \in C K_{n}$ has no bonds as a cylindrical king, thus there are $n$ distinct permutations $\sigma^{\prime} \in S_{n-1}$ such that $\sigma^{\prime} \prec \sigma$. We show now that each one of them is a cylindrical king permutation. According to Proposition 6.1, omitting a single entry from a permutation may decrease the cyclic breadth by at most one. Thus, each $\sigma^{\prime} \in S_{n-1}$ such that $\sigma^{\prime} \prec \sigma$ satisfies $\operatorname{cbr}\left(\sigma^{\prime}\right) \geq 3$ and by Observation $6.2, \sigma^{\prime} \in C K_{n-1}$.

Recall that a permutation $\sigma \in S_{n}$ is $k$-prolific if each $(n-k)$-subset of entries of $\sigma$ forms a unique pattern. In other words, $\sigma$ is $k$-prolific if it contains the maximal possible number of distinct permutations $\pi \in S_{n-k}$ such that $\pi \prec \sigma$. The breadth of a permutation affects its being $k$-prolific as shown in Theorem 2.25 in [2]: $\sigma$ is $k$-prolific if and only if $\operatorname{br}(\sigma) \geq k+2$. This idea can be extended to the poset of king permutations as well as to the poset of cylindrical king permutations. This is the motivation of the following definition.

Definition 6.3. We say that a permutation $\sigma \in K_{n}$, (resp. $\left.C K_{n}\right)$ is k-prolific in $K_{n}$, (resp. $C K_{n}$ ) if each $(n-k)$-subset of the entries of $\sigma=\sigma_{1} \cdots \sigma_{n}$ forms a unique pattern in $K_{n-k}$, (resp. $\left.C K_{n-k}\right)$.

Using the above definition, we prove the following theorem.

Theorem 6.1. A permutation $\sigma$ is $k$-prolific in $K_{n}$ if and only if $\operatorname{br}(\sigma) \geq k+3$.

Proof. Assume to the contrary that $\sigma$ is $k$-prolific but $b r(\sigma)<k+3$. This means that there are $1 \leq i, j \leq n$ such that $d_{\sigma}(i, j)<k+3$. For the sake of convenience, we assume that $i<j$ and $\sigma_{i}<\sigma_{j}$.

Now, let

$$
S=\left\{\sigma_{m} \mid i<m<j \text { or } \sigma_{i}<\sigma_{m}<\sigma_{j}\right\} .
$$

Obviously, $|S| \leq j-i-1+\sigma_{j}-\sigma_{i}-1$ so that $|S|+2 \leq d_{\sigma}(i, j)<k+3$ and thus $|S| \leq k$. Omitting the elements of $S$ from $\sigma$, we get a permutation $\sigma^{\prime} \in S_{n-|s|}$ such that $\sigma^{\prime} \prec \sigma$ and $\sigma^{\prime}$ contains a pair $u<v$ such that $d_{\sigma^{\prime}}(u, v)=2$, i.e. $\left(\sigma_{u}, \sigma_{v}\right)$ is a bond. For example, let $\sigma=\mathbf{9 7 2 4 1 5 8 3 6}$ and let $i=1$ and $j=4$. Then we have $S=\{7,2,5,6,8\}$ and after omitting the elements of $S$, we get $\sigma^{\prime}=\mathbf{4 3 1 2}$ which indeed has a bond.

Now, if $|S|=k$, this means that $\sigma^{\prime} \notin K_{n-k}$ so that $\sigma$ can not be $k$-prolific in $K_{n}$. If $|S|<k$ then we can omit from $\sigma^{\prime}$ a set of $k-|S|$ elements which does not contain the bond mentioned above and get an $(n-k)$-subset of the entries of $\sigma$ which creates a descendant of $\sigma$ which is not a member of $K_{n-k}$, so again $\sigma$ can not be $k$-prolific.

On the other hand, assume that $\operatorname{br}(\sigma) \geq k+3$ and we prove that $\sigma$ is $k$-prolific in $K_{n}$. First, by Theorem 2.25 of [2], $b r(\sigma) \geq k+2$ if and only if $\sigma$ is $k$-prolific in $S_{n}$, so that each omission of a $k$-set from $\sigma$ yields a different permutation in $S_{n-k}$. To show that each such $\pi \in S_{n-k}$ is indeed a king permutation, note that by Proposition 2.24 of [2], omitting a single entry from a permutation may decrease the breadth by at most one. Thus, omitting each $k$-set of elements forms a permutation $\pi$ with $b r(\pi) \geq 3$ which implies that $\pi \in K_{n-k}$.

The following theorem is the generalization of Theorem 6.1 for the poset of cylindrical king permutations.

Theorem 6.2. A permutation $\sigma$ is $k$-prolific in $C K_{n}$ if and only if $\operatorname{cbr}(\sigma) \geq k+3$. 
The proof is almost identical to the proof of Theorem 6.1 except for some necessary changes. Instead of the set $S$, we define the set:

$$
S_{\text {cyclic }}=\left\{\sigma_{m} \mid m \in((i, j)) \text { or } \sigma_{i}<\sigma_{m}<\sigma_{j}\right\} .
$$

Then we replace the breadth $b r$ by its cyclic parallel breadth $c b r$. At the end of the proof, we use Proposition 6.1 of this article instead of Proposition 2.24 of [2].

\section{The structure of the poset of cylindrical king permu- tation}

It is easy to observe that for $\sigma \in S_{n}$, and a regular bond $(a, a+1)$ in $\sigma$, either omitting $a$ or omitting $a+1$ gives the same permutation. In other words

$$
\nabla_{a}^{*}(\sigma)=\nabla_{a+1}^{*}(\sigma) .
$$

Example 7.1. For $\sigma=523641, \nabla_{2}^{*}(\sigma)=\nabla_{3}^{*}(\sigma)=42531$.

This enables one to count for each permutation $\sigma \in S_{n}$, the number of permutations $\pi \in S_{n-1}$ such that $\pi \prec \sigma$.

Unfortunately, Equation (10) does not hold anymore when we deal with edge bonds. For example, if $\sigma=246351$, then $\nabla_{2}^{*}(\sigma)=35241$ while $\nabla_{1}^{*}(\sigma)=13524$. In order to overcome this problem, we consider circular permutations. In order to do that, we define an equivalence relation based on considering each permutation as if it was written on a circle and not on a line. For example, according to this new view, the permutations 35241 and 13524 are considered identical. We formally address this identification in the next definition.

Definition 7.1. Recall that $C_{n}$ is the sub-group of $S_{n}$ generated by the cycle $\omega=(1 \cdots n)$. Define an equivalence relation on $S_{n}$ by $\sigma \sim \tau$ if and only if there is some $1 \leq i \leq n$ such that $\tau=\sigma \cdot \omega^{i}$ and let $S_{n} / C_{n}$ be the quotient space. Also, let us denote by $\operatorname{orb}(\sigma)$ the equivalence class of $\sigma$.

Example 7.2. Let $\sigma=23154 \in S_{5}$. Then

$$
\operatorname{orb}(\sigma)=\{23154,31542,15423,54231,42315\} .
$$

We now aim to characterize the permutations that serve as building blocks for the poset of cylindrical king permutations.

Recall from Equation (1) that, if $\sigma \sim \tau$ then $\sigma$ and $\tau$ have the same number of cyclic bonds. Therefore, $\sigma \in C K_{n}$ if and only if $\tau \in C K_{n}$.

In the next theorems we use the concept of cyclic separators, the definition of which we give now: In our recent paper [4], we defined a new concept, called separator. A separator in a permutation $\sigma=\sigma_{1} \cdots \sigma_{n} \in S_{n}$ is an element $\sigma_{i}$ whose omission forms a new bond. In particular, if $\sigma \in K_{n}$ is a king permutation, then the permutation obtained by the omission of $\sigma_{i}$ is a king permutation if and only if $\sigma_{i}$ is not a separator in $\sigma$. In order to characterize the structure of the poset of cylindrical king permutations, we need a cyclic version of that concept. We start by recalling the definition of the (regular) separator (see Definition 2.1 in [4]).

Definition 7.2. For $\sigma=\sigma_{1} \cdots \sigma_{n} \in S_{n}$ we say that $\sigma_{i}=a$ separates $\sigma_{j_{1}}$ from $\sigma_{j_{2}}$ in $\sigma$ if by omitting the element a from $\sigma$ we get a new bond. This happens if and only if one of the following cases holds:

1. $j_{1}, i, j_{2}$ are subsequent numbers and $\left|\sigma_{j_{1}}-\sigma_{j_{2}}\right|=1$, i.e

$$
\sigma=\ldots, b, a=\sigma_{i}, b \pm 1, \ldots
$$

In this case we say that a is a vertical separator.

2. $\sigma_{j_{1}}, \sigma_{i}=a, \sigma_{j_{2}}$ is an increasing or decreasing sub sequence of subsequent numbers, and $\left|j_{1}-j_{2}\right|=1$, i.e,

$$
\sigma=\ldots, a, \ldots, a \pm 1, a \mp 1, \ldots
$$

or

$$
\sigma=\ldots, a \pm 1, a \mp 1, \ldots, a, \ldots
$$

In this case we say that a is a horizontal separator.

Cylindrical king permutations are actually permutations having no cyclic bonds. In order to be able to deal with edge bonds, we have to modify the Definition 7.2 of the separator. 
Definition 7.3. Let $\sigma=\sigma_{1} \cdots \sigma_{n} \in S_{n}$. An element $\sigma_{i}$ will be called an edge separator if omitting it produces a new edge bond. This happens when one of the following holds:

1. If $\left|\sigma_{1}-\sigma_{n-1}\right|=1$ then $\sigma_{n}$ is a (vertical) edge separator.

2. If $\left|\sigma_{n}-\sigma_{2}\right|=1$ then $\sigma_{1}$ is a (vertical) edge separator.

3. If for some $1<i<n$ we have that $\sigma_{1}, \sigma_{i}, \sigma_{n}$ is an increasing or decreasing sub sequence of subsequent numbers, then we say that $\sigma_{i}$ is a (horizontal) edge separator.

We say that an element $\sigma_{i}$ is a cyclic separator if it is a regular or an edge separator. In this case, we say that $\sigma_{i}$ cyclically separates some element from another.

Example 7.3. Let $\sigma=52341$, then $\sigma_{5}=1$ is a (vertical) edge separator in $\sigma$. Let $\sigma=6257134$, then $\sigma_{3}=5$ is a (horizontal) edge separator in $\sigma$. If $\sigma=246135$, then $\sigma_{6}=5$ is a vertical edge separator and a horizontal (regular) separator in $\sigma$.

Theorem 7.1. For every $\sigma \in C K_{n}(n \geq 5)$ there is some $\pi \in \operatorname{orb}(31425) \cup \operatorname{orb}(24135)$ such that $\pi \preceq \sigma$.

Proof. We prove by induction on $n$, the case $n=5$ being trivial, since $C K_{5}=\operatorname{orb}(31425) \cup \operatorname{orb}(24135)$. We assume that each cylindrical king permutation of order $n-1$ contains at least one of the permutations of $\operatorname{orb}(31425) \cup \operatorname{orb}(24135)$ and prove that each cylindrical king permutation of order $n$ contains at least one element of this set.

Let $n>5$ and assume to the contrary that there is $\sigma=\sigma_{1} \cdots \sigma_{n} \in C K_{n}$ which does not contain any of the permutations in $\operatorname{orb}(31425) \cup \operatorname{orb}(24135)$. Then for every $1 \leq i \leq n$, the permutation $\nabla_{i}^{*}(\sigma)$, contains neither of them as well. By the induction hypothesis, $\nabla_{i}^{*}(\sigma) \notin C K_{n-1}$, which implies, that every element of $\sigma$ is a cyclic separator. In particular, 1 is a cyclic vertical separator. Hence, we have: $\sigma \in \operatorname{orb}(\ldots, a, 1, a+1, \ldots)$ or $\sigma \in \operatorname{orb}(\ldots, a+1,1, a, \ldots)$. Since $\sigma \in C K_{n}$, we know that $a>2$. Without loss of generality, assume that $\sigma \in \operatorname{orb}(\ldots, a, 1, a+1, \ldots)$. By the above, the element $a+1$ is a cyclic separator of $\sigma$. We have now two options as $a+1$ is horizontal or vertical:

- If $a+1$ is horizontal then $\sigma \in \operatorname{orb}(\ldots a+2, a, 1, a+1, \ldots)$. For the same reasons as above, $a$ is also a cyclic horizontal separator of $\sigma$. Thus $\sigma \in \operatorname{orb}(\ldots a+2, a, 1, a+1, a-1 \ldots)$. But the sub sequence $a+2, a, 1, a+1, a-1$ is a pattern which is isomorphic to 53142 , so that $\sigma$ contains an element of orb(31425).

- If $a+1$ is vertical then $\sigma \in \operatorname{orb}(\ldots a, 1, a+1,2 \ldots)$. As we saw above, $a$ is also a cyclic separator of $\sigma$ which must be horizontal so that $a=3$. This implies that $\sigma \in \operatorname{orb}(\ldots 3,1,4,2 \ldots)$. Since the element 5 has to be somewhere in $\sigma$, we must have $\sigma \in \operatorname{orb}(\ldots, 3,1,4,2, \ldots, 5, \ldots)$ which contains the pattern 31425 .

In Proposition 3.14 of [5], we proved that for each two king permutations $\sigma \in K_{n}$ and $\pi \in K_{n-2}$ such that $\pi \prec \sigma$ there is a king permutation $\tau$ such that $\pi \prec \tau \prec \sigma$. This does not hold for cylindrical king permutations. For example, for $\sigma=18463527 \in C K_{8}$ and $\pi=635241 \in C K_{6}$, even though $\pi \prec \sigma$, there is no $\tau \in C K_{7}$ such that $\pi \prec \tau \prec \sigma$.

Moreover, in Theorem 3.15 of [5] we claimed that if $\sigma \in K_{n}, \pi \in K_{m}$ such that $\pi \prec \sigma$ and $n-m>3$ then there exists a king permutation $\tau \in K_{k}$ such that $\pi \prec \tau \prec \sigma$ and $m-k \in\{1,3\}$. This implies that for every $\pi \in K_{m}$ and $\sigma \in K_{n}$ such that $\sigma$ covers $\pi$ in the poset $\mathbb{K}$, one has $n-m \leq 3$. This property does not hold true anymore in the poset $\mathbb{C K}$ as the following example verifies.

Example 7.4. Let $\sigma=579683142$ and $\pi=13524$ which is obtained from $\sigma$ by removing the elements $3,1,4,2$. It is easy to observe that there is no $\tau \in \mathbb{C K}$ such that $\pi \prec \tau \prec \sigma$.

We claim now that if $\sigma \in C K_{n}$ covers $\pi \in C K_{m}$ in the poset $\mathbb{C K}$, then $n-m \leq 4$.

Theorem 7.2. Let $\sigma \in C K_{n}$ and $\pi \in C K_{m}$ be such that $\pi \prec \sigma$ and $n-m>4$. Then there is some $\tau \in \mathbb{C K}$ such that $\pi \prec \tau \prec \sigma$.

The proof of Theorem 7.2 is very subtle and messy. It consists of many similar sub-cases which recur over and over. In order not to be too exhaustive, we present some basic observations and lemmas and then show how to deal with a few typical sub-cases and leave the rest of the proof for the reader. For the sake of simplifying the proof, we present a series of claims.

Observation 7.1. Let $\sigma$ be a permutation, let $\pi \in \mathbb{C K}$, and let $i$ be such that $\pi \prec \nabla_{i}^{*}(\sigma)$. If $\nabla_{i}^{*}(\sigma)$ contains the cyclic bond consisting of the elements $j$ and $k$ (of $\sigma$ ) then $\pi \prec \nabla_{k}^{*}(\sigma)$ or $\pi \prec \nabla_{j}^{*}(\sigma)$,

Moreover, if the bond is not an edge bond then both $\pi \prec \nabla_{k}^{*}(\sigma)$ and $\pi \prec \nabla_{j}^{*}(\sigma)$. 
Example 7.5. In the left picture of Figure 2, $\sigma=264159837 \in S_{9}, \pi=241635 \in C K_{6}$, such that $\pi \prec \sigma$, $\pi \prec \nabla_{5}^{*}(\sigma)$. In $\nabla_{5}^{*}(\sigma)=25418736$ there is a regular bond $(5,4)$ (the elements 4 and 6 in $\sigma$ ), we get that $\pi \prec \nabla_{4}^{*}(\sigma)$ and $\pi \prec \nabla_{6}^{*}(\sigma)$.

In the right picture $\sigma=264159837 \in S_{9}, \pi=253146 \in C K_{6}$, such that $\pi \prec \sigma, \pi \prec \nabla_{7}^{*}(\sigma)$. In $\nabla_{7}^{*}(\sigma)=$ 26415873 there is an edge bond $(2,3)$ (the elements 2 and 3 in $\sigma)$, we get that $\pi \prec \nabla_{3}^{*}(\sigma)$ but $\pi \nprec \nabla_{2}^{*}(\sigma)$.

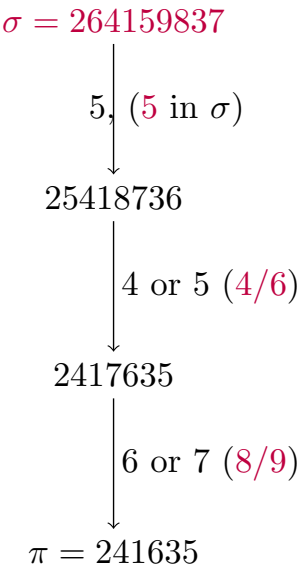

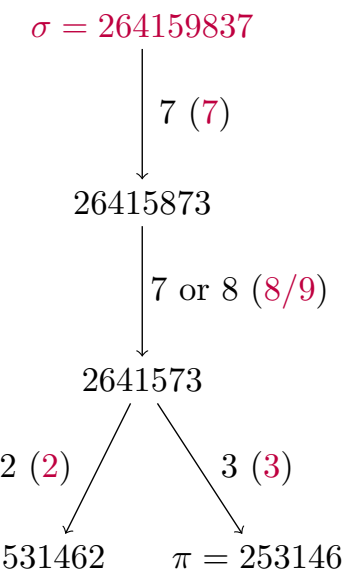

Figure 2:

Definition 7.4. Let us denote by $\nabla_{A}^{*}(\sigma)$ the permutation $\nabla_{i_{1}}^{*}\left(\nabla_{i_{2}}^{*}\left(\ldots\left(\nabla_{i_{k}}^{*}(\sigma)\right)\right)\right.$ where $A=\left\{i_{1}, \ldots, i_{n}\right\}$ and $i_{1}<\cdots<i_{k}$. For example $\sigma=264159837 \in S_{9}, \pi=241635 \in C K_{6}$, so that $\pi \prec \sigma$. If we let $A_{1}=$ $\{4,5,8\}, A_{2}=\{4,5,9\}, A_{3}=\{5,6,8\}, A_{4}=\{5,6,9\}$, we get $\pi=\nabla_{A_{i}}^{*}(\sigma)$ for $1 \leq i \leq 4$.

In the following observation, when we write 'adjacent', we take it in the cyclic meaning, i.e. in $a_{1} \cdots a_{n}$, the elements $a_{1}$ and $a_{n}$ are considered here as adjacent.

Observation 7.2. Let $\sigma \in \mathbb{C K}$ be such that $\sigma$ contains the (cyclic) block $(a+2, a+4, a+1, a+3)$ or its reverse.

1. If we remove any single element $i$ from the block $(a+2, a+4, a+1, a+3)$ in $\sigma$ then $\nabla_{i}^{*}(\sigma)$ will contain a cyclic bond. Moreover, we will not obtain a cylindrical king permutation out of $\nabla_{i}^{*}(\sigma)$ unless we omit at least 3 elements from the block, in which case, it contracts to the element $a+1$.

2. If the elements $a$ and $a+5$ are not placed adjacent to the block $(a+2, a+4, a+1, a+3)$ or its reverse in $\sigma$, then by omitting any 3 elements of the block we get a cylindrical king permutation.

For example, let $\sigma=391426857$ with the block $(6,8,5,7)$. Note that $\tau=\nabla_{7}^{*}(\sigma)=38142675$ which contains the bond $(6,7), \nabla_{6}^{*}(\tau)=3714265$ with the bond $(6,5)$, but $\nabla_{\{5,6,7\}}^{*}(\sigma)=361425$ which is a cylindrical king permutation.

3. If $b \in\{a, a+5\}$ is placed adjacent to the block $(a+2, a+4, a+1, a+3)$ or its reverse in $\sigma$, then by removing either $b$ or the entire block we get a cylindrical king permutation. This is due to the fact that in this case $a$ and $a+5$ can not be cyclic separators of any type in $\sigma$. For example, let $\sigma=319246857$ with the block $(6,8,5,7)$. Note that $\tau=\nabla_{4}^{*}(\sigma)=31825746 \in C K_{8}$.

Lemma 7.1. Let $\sigma \in C K_{n}, \pi \in C K_{m}$ be such that $\pi \prec \sigma$ and $n-m>4$. Assume that

- There is some element $a \in \mathbb{N}$ such that $\sigma \in \operatorname{orb}(\ldots, a+2, a+4, a+1, a+3, \ldots)$ or $\sigma \in \operatorname{orb}(\ldots, a+3, a+$ $1, a+4, a+2, \ldots)$.

- There is some $1 \leq i \leq 4$ such that $\pi \prec \nabla_{a+i}^{*}(\sigma)$.

Then there is some $\tau \in C K_{k}$ such that $\pi \prec \tau \prec \sigma$ and $n-k \leq 4$.

Proof. Assume without loss of generality that $\pi \prec \nabla_{a+2}^{*}(\sigma)$ and that $\sigma$ contains the block $(a+2, a+4, a+1, a+3)$. The proof depends on the location of this block in $\sigma$. The possibilities are: $\sigma=\ldots, a+2, a+4, a+1, a+3$ or $\sigma=a+2, a+4, a+1, a+3, \ldots$ or $\sigma=\ldots, a+2, a+4, a+1, a+3, \ldots$ or $\sigma=a+1, a+3, \ldots, a+2, a+4$ or $\sigma=a+3, \ldots, a+2, a+4, a+1$ or $\sigma=a+4, a+1, a+3, \ldots, a+2$.

Here we show only the first case: $\sigma=\ldots, a+2, a+4, a+1, a+3$ which seems to be the most general case in the sense that it contains all the arguments we need. The other cases can be proved using the same method.

According to Observations 7.1 and 7.2.1, $\pi \prec \nabla_{A}^{*}(\sigma)$ for $A=\{a+2, a+3, a+4\}$. If $\nabla_{A}^{*}(\sigma) \in \mathbb{C K}$ then we are done (just take $\left.\tau=\nabla_{A}^{*}(\sigma)=\ldots, a+1\right)$. Otherwise, according to 7.2.2, either $a$ or $a+5$ is adjacent to the block $(a+2, a+4, a+1, a+3)$. Assume without loss of generality. that $a$ is adjacent to the above block. We have 2 possibilities. 
- If $\sigma=\ldots, a, a+2, a+4, a+1, a+3$ then $\nabla_{A}^{*}(\sigma)=\ldots, a, a+1$ and according to Observation 7.1, $\pi \prec \nabla_{a}^{*}\left(\nabla_{A}^{*}(\sigma)\right)=\nabla_{A^{\prime}}^{*}\left(\nabla_{a}^{*}(\sigma)\right) \prec \nabla_{a}^{*}(\sigma)$ for $A^{\prime}=\{a+1, a+2, a+3\}$ and so by $7.2 .3 \tau=\nabla_{a}^{*}(\sigma)$ and we are done.

- If $\sigma=a \cdots b, a+2, a+4, a+1, a+3$ (where $b \neq a+5$ ) then $\nabla_{A}^{*}(\sigma)=a \cdots a+1$. By Observation 7.1, $\pi \prec \nabla_{a}^{*}\left(\nabla_{A}^{*}(\sigma)\right) \prec \nabla_{a}^{*}(\sigma)$ or $\pi \prec \nabla_{B}^{*}(\sigma)$ for $B=A \cup\{a+1\}$. We divide in two cases, depending on whether $\pi \prec \nabla_{a}^{*}(\sigma)$ or not.

1. If $\pi \prec \nabla_{a}^{*}(\sigma)$ then we are done since according to 7.2.3., $\tau=\nabla_{a}^{*}(\sigma)$ is a cylindrical king permutation.

2. If $\pi \nprec \nabla_{a}^{*}(\sigma)$ then if $\nabla_{B}^{*}(\sigma)$ is a cylindrical king permutation then we are done by taking $\tau=\nabla_{B}^{*}(\sigma)$. (Note that this is the single case in which $n-k=4$ ). Otherwise, the permutation $\nabla_{B}^{*}(\sigma)$ contains a cyclic bond, leading to one of the following two possibilities:

(a) We have $\sigma=a, a+5, \ldots, a+2, a+4, a+1, a+3$ which is impossible since then $\nabla_{B}^{*}(\sigma)$ is of the form $a, a+1, \ldots$ and by 7.1 we get $\pi \prec \nabla_{a}^{*}(\sigma)$ which contradicts the assumption.

(b) We have $\sigma=a, \ldots, a-1, a+2, a+4, a+1, a+3$ and according to Observation 7.1, $\pi \prec$ $\nabla_{a-1}^{*}\left(\nabla_{B}^{*}(\sigma)\right) \prec \nabla_{a-1}^{*}(\sigma)$. We claim that $\nabla_{a-1}^{*}(\sigma)$ is a cylindrical king permutation. Indeed, otherwise $\sigma=a, a-2 \ldots, a-1, a+2, a+4, a+1, a+3$ and $\nabla_{a-1}^{*}(\sigma)$ contains a regular bond consisting of the elements $a$ so that $a-2$ of $\sigma$. Hence, due to 7.1, $\pi \prec \nabla_{a}^{*}(\sigma)$ which contradicts the assumption. Now, we can take $\tau=\nabla_{a-1}^{*}(\sigma)$ and we are done.

Now we return to the proof of Theorem 7.2. Let $\sigma \in C K_{n}, \pi \in C K_{m}$ be such that $\pi \prec \sigma$, and $n-m>4$ and let us prove that there is $\tau \in C K_{k}$ such that $\pi \prec \tau \prec \sigma$ and $n-k \leq 4$.

Proof of Theorem 7.2. Since $\mathbb{C K} \subset \mathbb{K}$, we have that $\pi \in K_{m}$ and $\sigma \in K_{n}$. Thus, according to Theorem 3.15 in [5], there is $\tau \in K_{k}$ such that $\pi \prec \tau \prec \sigma$ and $n-k \in\{1,3\}$. If $n-k=3$ then according to the proof of Theorem 3.15 in [5], $\sigma$ contains the block $(a+2, a+4, a+1, a+3)$ or its reverse and $\pi$ is obtained from $\sigma$ by omitting 3 elements from this block. This means that the conditions of Lemma 7.1 hold and so our claim is proved.

Assume now that $n-k=1$. If $\tau \in \mathbb{C K}$ then we are done. Otherwise, $\tau=\nabla_{b}^{*}(\sigma)=a \cdots a+1$, for some edge separator $b$ of $\sigma$. If $b$ is a vertical separator, then without loss of generality, we assume that $b>a+1$ and we have $\sigma=a, \ldots, a+1, b$. Else $b$ is a horizontal separator and so $\sigma=a, \ldots, b=a+1, \ldots, a+2$ (or their reverses). Here we consider only the vertical case: $\sigma=a, \cdots, a+1, b$. Since $\pi \prec \nabla_{b}^{*}(\sigma)=a, \ldots, a+1$, by Observation $7.1, \pi \prec \nabla_{a}^{*}(\sigma)$ or $\pi \prec \nabla_{a+1}^{*}(\sigma)$.

We consider here only the case $\pi \prec \nabla_{a+1}^{*}(\sigma)$ but $\pi \nprec \nabla_{a}^{*}(\sigma)$. If $\nabla_{a+1}^{*}(\sigma) \in \mathbb{C} \mathbb{K}$ then we are done. Otherwise $\nabla_{a+1}^{*}(\sigma)$ contains a cyclic bond. Thus we have for $\sigma$ one of the following options: either $\sigma=a, \ldots, b \pm 1, a+1, b$ or $\sigma=a, a+2 \ldots, a+1, b$. Let us consider again the first case, $\sigma=a, \ldots, b+1, a+1, b$. In this case, $\nabla_{a+1}^{*}(\sigma)$ has a regular bond consisting of the elements $b$ and $b+1$ of $\sigma$ and so by Observation 7.1, $\pi \prec \nabla_{b}^{*}(\sigma)$ and $\pi \prec \nabla_{b+1}^{*}(\sigma)$. If $\nabla_{b+1}^{*}(\sigma)$ is a cylindrical king permutation, we are done. Otherwise, the omission of the element $b+1$ from $\sigma$ produces a cyclic bond. Again, there are two options for the structure of $\sigma$. Either $a=b+2$ and thus $\sigma=a, \ldots, a-1, a+1, a-2$, so by Lemma 7.1 we are done, or (if $a \neq b+2) \sigma=a, \ldots, a+2, b+1, a+1, b$. In this case $\nabla_{A}^{*}(\sigma)=a, \ldots, a+1, b$ for $A=\{a+2, b+1\}$ is a cylindrical king permutation. (Indeed, the pair $(a+1, b)$ is not a bond since $a+1$ and $b$ are adjacent in $\sigma$. The elements $a+1$ or $a-1$ can not appear next to the element $a$ since $\sigma$ is a cylindrical king permutation). This completes the proof.

\section{Directions for further research}

- In [2], Bevan, Homberger and Tenner proved that a $k$-prolific permutation in $S_{n}$ must be at least of size $\frac{k^{2}}{2}+2 k+1$. A challenge may be to find a lower bound for the size of a $k$-prolific permutation in $K_{n}$ and in $C K_{n}$.

- In the chess game, one can further enable the pieces also to go off a column and reappear at the beginning of that column, thus obtaining a 'chess on the torus'. This variant is counted by OEIS A089222. The permutations describing non-attacking kings on this board are called toric king permutations and constitute a sub-poset of their own. The work on these permutations is in progress.

\section{Acknowledgments}

The authors want to thank Yuval Roichman for fruitful discussions. 


\section{References}

[1] M. Abramson and W. O. J. Moser, Permutations without rising or falling w-sequences, Ann. Math. Statist. $38: 4$ (1967), 1245-1254.

[2] D. Bevan, C. Homberger, and B. E. Tenner, Prolific permutations and permuted packings: downsets containing many large patterns, J. Combin. Theory, Ser. A 153 (2018), 98-121.

[3] B. Aspvall and F. Liang, The dinner table problem, Stanford Department of Computer Science Report, No. STAN-(X-80-829), 1980.

[4] E. Bagno, E. Eisenberg, S. Reches, and M. Sigron, On the Sparseness of the Downsets of Permutations via Their Number of Separators, Enumer. Combin. Appl. 1:3 (2021), Article \#S2R21.

[5] E. Bagno, E. Eisenberg, S. Reches, and M. Sigron, On the poset of King-Non-Attacking permutations, European J. Combin. 87 (2020), 103119.

[6] D. Bevan, Permutation patterns: Basic Definitions and Notations, The open University, https://arxiv. org/pdf/1506.06673.pdf, 2015.

[7] S.R. Blackburn, C. Homberger, and P. Winkler, The minimum Manhattan distance and minimum jump of permutations, J. Combin. Theory, Ser. A 161 (2019), 364-386.

[8] P. Cellini, Cyclic Eulerian Elements, European J. Combin. 19:5 (1998) 545-552.

[9] M. Coleman, An Answer to a Question by Wilf on Packing Distinct Patterns in a Permutation, Electron. J. Combin. 11:1 (2004), Note 8.

[10] P. Flajolet and R. Sedgewick, Analytic combinatorics, Cambridge University Press, Cambridge, 2009.

[11] C. Homberger, Counting Fixed-Length Permutation Patterns, Online J. Anal. Comb. No. 7 (2012).

[12] G. Jelliss, Cylinder Chess, Variant Chess 3:22 (1996), 32-33.

[13] V. Kotěšovec, Non-attacking chess pieces, chess and mathematics, 2010.

[14] T. K. Petersen, Cyclic Descents and P-Partitions, J. Alg. Combin. 22 (2005), 343-375.

[15] D. P. Robbins, The probability that neighbors remain neighbors after random rearrangements, Amer. Math. Monthly 87:2 (1980), 122-124.

[16] N. J. A. Sloane, Sequence A002464 in "The On-Line Encyclopedia of Integer Sequences", https://oeis . org/A002464, 1964.

[17] N. J. A. Sloane, Sequence A002493 in "The On-Line Encyclopedia of Integer Sequences", https://oeis. org/A002493, 1964.

[18] R. Tauraso, The dinner table problem, the rectangular case, Integers 6 (2006), A11. 